\title{
Effect of DIC spatial resolution, noise and interpolation error on identification results with the VFM
}

\author{
Marco Rossi ${ }^{\mathrm{a}}$, Pascal Lava ${ }^{\mathrm{b}}$, Fabrice Pierron $^{\mathrm{c}}$, Dimitri Debruyne ${ }^{\mathrm{b}}$, Marco \\ Sasso $^{\mathrm{a}}$ \\ ${ }^{a}$ Università Politecnica delle Marche, Monte Dago, via Brecce Bianche, 60131 Ancona, \\ Italy.E-mail:m.rossi@univpm.it \\ ${ }^{b}$ Department MTM, Katholieke Universiteit Leuven, Kasteelpark Arenberg 44, B-3001 \\ Leuven (Heverlee) \\ ${ }^{c}$ Faculty of Engineering and the Environment, University of Southampton, Highfield, \\ Southampton SO17 1BJ, UK
}

\begin{abstract}
The use of experimental tests which involve full-field measurements to characterize mechanical material properties is becoming more widespread within the engineering community. In particular Digital Image Correlation (DIC) on white light speckles is one of the most used tools, thanks to the relatively low cost of the equipment and the availability of dedicated software. Nonetheless the impact of measurement errors on the identified parameters is still not completely understood. To this purpose, in this paper, a simulator able to numerically simulate an experimental test which involves DIC is presented. The chosen test is the Unnotched Iosipescu (UI) test used to identify the orthotropic elastic parameters of composites. Synthetic images are generated and then analysed by DIC. Eventually the obtained strain maps are used to identify the elastic parameters with the Virtual Fields Method (VFM). The numerical errors propagating through the simulation procedure are carefully characterized. Besides, the simulator is used to compare the performances of DIC and the grid method in the identification process with the VFM. Finally, the influence of DIC settings on the identification error
\end{abstract}


is studied as a function of the camera digital noise level, in order to find the best testing configuration.

Keywords:

Digital image correlation, Virtual fields method, Error assessment, Simulated experiments

\section{Introduction}

Material characterization is increasingly performed with the aid of fullfield measurements. Indeed, contrary to standard mechanical tests, these can be designed in order to produce a heterogeneous stress-strain field inside the specimen. In this way, a single experiment can yield a wealth of information since the material is loaded under different stress conditions at different points of the same specimen. A standard test, instead, results in a uniform state of stress, and accordingly many experiments are required to identify for instance orthotropic material parameters.

Within the class of full-field measurements digital image correlation (DIC) plays nowadays a very prominent role [1]. Indeed, thanks to its high flexibility at various levels of deformation for various kinds of materials, DIC has become a standard measurement tool in most of mechanical labs.

Nowadays many methods are available to extract the mechanical properties of materials from full-field measurements. Some of them rely on finite element updating [2-6], others on the constitutive equation gap method [7,8], the equilibrium gap method [9] or the reciprocity gap method [10]. In the presented paper the Virtual Fields Method (VFM) [11] has been adopted. The VFM allows to extract the material properties from the measured strain field using the global equilibrium equation written in terms of the principle of 
virtual work. This method has been successfully employed in many different branches of mechanics, e.g. vibration [12] or elasto-plasticity [13, 14], and for many different materials; for instance, it was recently used to evaluate the properties of 3D printed materials [15].

In spite of such extensive research on the identification techniques, very limited work can be found on the coupling between the measurement technique, like DIC, and the inverse identification method, like the VFM. A first attempt in this direction was made by Pierron et al. [16], who used the sensitivity to noise of VFM to evaluate the best specimen configuration in the UI test.

On the other hand, DIC developers judge the performances of DIC programmes in terms of correlation error or computational time. A very exhaustive analysis was conducted by Bornert et al. [17], the error of DIC was assessed using simulated images and sinusoidal displacement fields. In that paper, different DIC packages have been compared and master curves were proposed to illustrate the influence of various parameters such as the subset size, the speckle size, the interpolation functions etc. Similar work has been performed by Lava et al. $[18,19]$ for the plastic deformation of materials based on FE displacement fields. In other studies, standardized experimental tests are employed to evaluate the DIC error instead of computer generated images [20, 21]. Wattrisse et al. [22] analysed the error induced by a strain localization during a tensile test on sheet metals. The uncertainty of DIC on heterogeneous strain fields was studied in [23]. The effect of the adopted shape functions was assessed in [24].

From this review, it turns out that, historically, the DIC errors and the identification errors have been treated separately. However, a strict connection exists between the optical technique, the experimental conditions used 
to measure the kinematic fields and the final outcome of the identification procedure. These two aspects should be studied at the same time, in particular for those cases where the spatial resolution is crucial, e.g. identification of defects, strain localizations, high deformation gradients. Knowledge of how the measurement technique influences the identification procedure would lead to design the experimental test as a function of the identification procedure, choosing the best geometry for the specimen, the optimal smoothing function etc. Accordingly, all the degrees of freedom in the identification chain can be controlled.

A possible strategy to tackle the problem is to numerically simulate the whole measurement chain. This approach was already presented by Rossi and Pierron [25] for the UI test [26]. In that paper, starting from a FE simulation, synthetic images were generated to reproduce the acquisition process of a CCD camera. Artificial noise was added to the synthetic images, then the grid method [27, 28], a full-field optical measurement technique, was used to extract the full-field displacement and strain fields. Finally the "measured"strain fields were used to identify the material properties using the VFM. Thanks to this approach, different test configurations were evaluated and critical aspects such as the effect of spatial resolution, the influence of smoothing and missing data, were quantitatively investigated.

In the present paper, a similar procedure is developed using DIC as optical full-field measurement technique. In this case, the reference image is a random white light speckle pattern which has to be numerically deformed. In contrast to the grid method, no analytical expression for the reference images exists, making the numerical deformation process of the synthetic images more cumbersome. The DIC method, on the other hand, is more frequently adopted since the bonding of the cross-hatch pattern on 
the specimen for grid testing is very hard. In addition, the measurement range of the grid method is more restricted.

Although numerical tools are available to generate artificial speckle patterns [29], real images of an actual speckle pattern have been used here. In this way, the complete identification procedure can be simulated starting from speckle patterns which are derived from actual experiments. The analysis is again focused on the UI test.

The aim of the paper is to investigate the coupling between DIC and identification with VFM, but, at the same time, it offers a critical analysis of the numerical method used to generate simulated experiments in order to highlight the limitations of this approach. The long term objective of this research is to provide a framework for uncertainty quantification when using heterogeneous tests, full-field measurements and inverse identification. This is an essential step if such procedures are to become test standards in the future.

\section{Theoretical background}

The identification procedure is composed of two techniques: DIC, that is the optical full-field method used to measure the displacement field at the surface of specimens during the test, and the VFM, which uses the measured strain fields to identify the material parameters. Both techniques are well established in the scientific community, for instance the reader can refer to [1] for DIC and [11] for the VFM. In this section a brief summary of the two methods is provided to ease the comprehension of the following parts and to point out some aspects that will be used later, like for instance the concept of virtual strain gauge. 


\subsection{Digital Image Correlation}

In a subset-based DIC (also denoted as "local approach") method, a matching between two speckle patterns is accomplished by considering a pixel and its neighbourhood in the undeformed image $f$ and searching for the same subset in the deformed image $g$, by minimization of a similarity criterion. In general, the origin of an $(x, y)$ coordinate system is located at the upper-left corner of $f(x, y)$. Let us denote $\left(x_{s c}, y_{s c}\right)$ the coordinates of the centre pixel of a $(2 N+1) \cdot(2 N+1)$ subset in image $f(x, y)$, with $N$ a positive integer number. A typical similarity criterion is the zero-mean normalized sum-of-squared-differences correlation coefficient $r_{Z N S S D}$, defined as

$$
\begin{aligned}
r_{Z N S S D}=\sum_{x} \sum_{y}\left[\frac{f[x, y]-\bar{f}}{\sqrt{\sum_{x} \sum_{y}(f[x, y]-\bar{f})^{2}}}-\right. & \left.\frac{g[x, y ; \mathbf{s}]-\bar{g}}{\sqrt{\sum_{x} \sum_{y}(g[x, y ; \mathbf{s}]-\bar{g})^{2}}}\right]^{2}
\end{aligned}
$$

with $\sum_{y}=\sum_{y=y_{s c}-N}^{y_{s c}+N}, \sum_{x}=\sum_{x=x_{s c}-N}^{x_{s c}+N}$ and $\bar{f}$ and $\bar{g}$ the mean values of the pixel intensities throughout the reference and deformed subset, respectively. This correlation criterion is insensitive to both offsets in illumination and contrast changes.

The unknown parameter vector $\mathbf{s}$ relates coordinates in the reference image $f$ to the corresponding coordinates in the deformed image $g$ via subset shape functions. The way in which the subset can deform during the correlation process is defined by the polynomial order of these shape functions and thus, the number of parameters in $\mathbf{s}$. The values of these parameters are obtained via an iterative Levenberg-Marquardt optimization algorithm, 
minimizing the correlation criterion in Eq.(1). This iterative process, however, involves the evaluation of grey-value and grey-value derivatives at noninteger pixel locations. Here, a bicubic spline algorithm is adopted.

This procedure allows to retrieve the displacement fields with one measurement point at the centre of each subset. At this point, the strain fields need to be calculated from the displacement with some care, since noise tends to get amplified by numerical differentiation. Many techniques can be used for this purpose, and the estimated displacement fields may be smoothed before the differentiation process.

In this work, an analytical expression of a surface is determined, which, in a least squares sense, approximates the displacement values in a selected region.

First, a square "strain window" is selected containing $N \times N$ discrete displacement data points in the vicinity of the point of interest $\left(x_{0}, y_{0}\right)$. Then, the displacements are analytically approximated by making use of Lagrange polynomials:

$$
\begin{aligned}
& u\left(x_{0}, y_{0}\right)=a_{i j}^{u} x_{0}^{i} y_{0}^{j} \\
& v\left(x_{0}, y_{0}\right)=a_{i j}^{v} x_{0}^{i} y_{0}^{j},
\end{aligned}
$$

where Einstein's summation convention is used and $i, j=0,1$ and $i, j=$ 0,1,2 correspond to bilinear (Q4) and biquadratic (Q9) Lagrange polynomials, respectively.

The expressions in Eq.(2) are linear in the unknown parameter sets $a_{i j}^{u}$ and $a_{i j}^{v}$. Accordingly, their determination is straightforward and the deformation gradient $\mathbf{F}$ can be easily derived as 


$$
\mathbf{F}^{\mathbf{Q 4}}=\left[\begin{array}{cc}
1+a_{10}^{u}+a_{11}^{u} y_{0} & a_{01}^{u}+a_{11}^{u} x_{0} \\
a_{10}^{v}+a_{11}^{v} y_{0} & 1+a_{01}^{v}+a_{11}^{v} x_{0}
\end{array}\right]
$$

A similar expression can be derived for the biquadratic case. The strain field is then derived from the deformation gradient $\mathbf{F}$ choosing the appropriate strain tensor (e.g. infinitesimal strain tensor, Green-Lagrange, logarithmic strain etc. ). In this case, the infinitesimal strain theory is adopted since the deformation is in the elastic range.

This process is such that the resulting strain field calculated at a given data point depends on a certain number of displacement data points around it. This can be considered as the 'gauge' size. Therefore it is useful to define a quantity that reflects the actual physical dimensions of the adopted gauge for the strain measurement.

This quantity will be denoted "virtual strain gauge" (VSG) further on. Together with the subset size, it is a regularization parameter that must be chosen with the highest care. The VSG has dimension of pixel and is defined as:

$$
\mathrm{VSG}=[(N-1) \times \text { step size }]+1
$$

where the step size is the distance in pixel between two subsequent subset windows and $N \times N$ are the discrete displacement points used to compute the strain tensor.

\subsection{The virtual fields method (VFM)}

The VFM is based on the principle of virtual work that, for a solid of any shape of volume $V$ and boundary surface $\partial V$, in the case of small perturbations and absence of body forces, can be written as: 


$$
\int_{V} \boldsymbol{\sigma}: \varepsilon^{*} d V=\int_{\partial V} \overline{\mathbf{T}} \cdot \mathbf{u}^{*} d S
$$

where $\boldsymbol{\sigma}$ is the stress vector, $\overline{\mathbf{T}}$ the surface forces acting at the boundary, $\mathbf{u}^{*}$ a continuous and differentiable virtual field, $\boldsymbol{\varepsilon}^{*}$ the corresponding virtual strain field and : and t the scalar products between 2nd order tensors and vectors, respectively. In the case of an in-plane test, if $t$ is the constant thickness of the volume $V$ and $S$ the planar surface, the problem reduces to a 2-D plane stress situation and Eq. 5 becomes:

$$
t \int_{S} \boldsymbol{\sigma}: \varepsilon^{*} d S=t \int_{\partial S} \overline{\mathbf{T}} \cdot \mathbf{u}^{*} d l
$$

The constitutive equation for linear orthotropic materials, using the conventional notation for contracted indices $x x \rightarrow x, y y \rightarrow y, x y \rightarrow s$, writes:

$$
\left(\begin{array}{c}
\sigma_{x} \\
\sigma_{y} \\
\sigma_{s}
\end{array}\right)=\left[\begin{array}{ccc}
Q_{x x} & Q_{x y} & 0 \\
Q_{x y} & Q_{y y} & 0 \\
0 & 0 & Q_{s s}
\end{array}\right]\left(\begin{array}{c}
\varepsilon_{x} \\
\varepsilon_{y} \\
\varepsilon_{s}
\end{array}\right)
$$

$\mathbf{Q}$ is the in-plane stiffness matrix and its four independent components are the parameters to be identified. The reference frame here is such that $x$ and $y$ coordinates correspond to the orthotropy axes, with $x$ parallel to the fibre direction. Assuming that the material is homogeneous and therefore $\mathbf{Q}$ is constant, the stress tensor in Eq. 6 can be rewritten in terms of the strain tensor using Eq. 7 and the constants moved out of the integrals: 


$$
\begin{aligned}
& Q_{x x} \int_{S} \varepsilon_{x} \varepsilon_{x}^{*} d S+Q_{y y} \int_{S} \varepsilon_{y} \varepsilon_{y}^{*} d S+ \\
& Q_{x y} \int_{S}\left(\varepsilon_{x} \varepsilon_{y}^{*}+\varepsilon_{y} \varepsilon_{x}^{*}\right) d S+Q_{s s} \int_{S} \varepsilon_{s} \varepsilon_{s}^{*} d S= \\
& \int_{\partial S} T_{x} u_{x}^{*} d l+\int_{\partial S} T_{y} u_{y}^{*} d l
\end{aligned}
$$

At this point, introducing four independent virtual fields in Eq. 8, four linear equations are obtained that can be used to identify directly the four unknown parameters $Q_{x x}, Q_{y y}, Q_{x y}$ and $Q_{s s}$. The strain components $\varepsilon_{x}$, $\varepsilon_{y}$ and $\varepsilon_{s}$ are measured on the specimen surface using a full-field optical technique, and in order to solve the system, the virtual displacements have to be chosen in such a way that the only information involved in the second term of Eq. 8 is the global load measured by the load cell of the test machine. Since a full-field optical technique provides a discrete description of the strain field, the integrals of Eq. 8 are approximated by discrete sums. In this case the required four independent virtual fields are automatically chosen using optimised virtual fields defined by polynomials. This is a standard procedure when the VFM is used to identify the elastic properties of materials, see [11].

\section{Simulation of the experimental test}

The simulated experiment is the unnotched Iosipescu (UI) test, which is a shear test on a rectangular specimen that produces an heterogeneous strain field. An example of the experimental set-up is given in Figure 1. This test has already been validated in several applications with the VFM (fibre composites, wood) and therefore it represents a good benchmark to perform error estimation of the complete identification procedure. 




Figure 1: Experimental set-up of the unnotched Iosipescu (UI) test.

The complete measurement chain for this UI test has been numerically simulated. The different steps are represented in Figure 2. The input parameters are the stiffness components, the design variables, i.e. the free length $L$ and the fibre orientation angle $\alpha$, and the CCD camera characteristics such as the pixel resolution, the dynamic range, the level of noise of the CCD sensor. An FE model of the test is built up with reference material input data that will serve as a benchmark later on. Then the displacement field computed by $\mathrm{FE}$ is interpolated in order to have a displacement value at each pixel position of the image. Two synthetic images are then generated (reference and deformed respectively) and grey level white noise is added to each image. From this point onwards, the procedure follows the same steps as in a real experimental test. DIC is applied to obtain the displacement and strain fields. Then the strain field and the applied force are input in the VFM algorithm to extract the stiffness parameters of Eq. 7. Finally, 
these derived material parameters can be compared to the reference material data of the FE model. Here, the comparison is carried out using an error function, defined later on.



Figure 2: Flow chart of the simulation process

Each step of the procedure is susceptible to introduce errors that will influence the final identification results. There are two types of error, the ones generated by the numerical procedure and the ones that reproduce actual experimental errors. The first type of error is an artefact that should be minimized. Because it is not possible to completely eliminate the numerical artefacts, it is important to assess the weight of such errors in the overall error. The various steps involved in Figure 2 are now detailed.

\subsection{FE model}

The FE model of the UI test was built up with ABAQUS Standard, the model is the same as described in [25] and is illustrated in Figure 3. Here it is used to obtain realistic displacement distributions of the UI test to be 


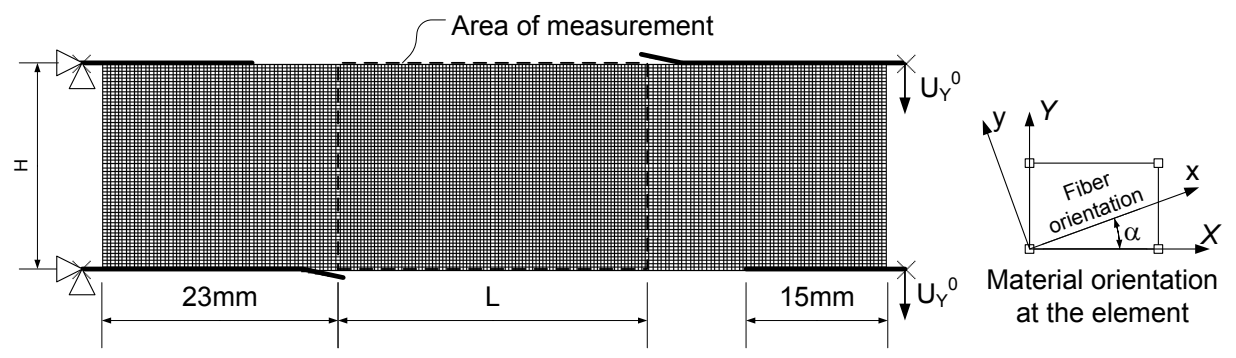

Figure 3: FEM model of the UI test, with boundary conditions and element material coordinate system [25].

used to deform the synthetic images. The model is parametric, two design variables can be varied by the user, the free length $L$ and the fibre orientation (orthotropy) angle $\alpha$. As illustrated in Figure 3, the material coordinate system is $(x, y)$ while the global coordinate system is $(X, Y)$. The analysis is linear elastic using the small strain formulation. The material model is orthotropic elasticity model that reproduces the behaviour of a glass/epoxy unidirectional composite. The constitutive parameters are, in this case, four independent stiffnesses, their numerical values are listed in Table 1.

For each configuration of $\alpha$ and $L$, different levels of force can be used to load the specimen. If the force is increased the measured displacements increase as well and, consequently, the signal-to-noise ratio increases with a beneficial effect on the identification. However the amount of force cannot be increased over a certain limit because of damage occurrence or deviation from linear elastic behaviour.

In order to keep such effect into consideration, the applied force was scaled so that the maximum stress inside the specimen guarantees linear elasticity. Because of the orthotropic behaviour of the material, different stress levels can be reached in different directions, in tension and in com- 


\begin{tabular}{cccc}
\hline \multicolumn{2}{c}{ Stiffness } & \multicolumn{2}{c}{ Maximum stress } \\
\hline$Q_{x x}(\mathrm{MPa})$ & 40920 & $S_{+x}(\mathrm{MPa})$ & 1000 \\
$Q_{y y}(\mathrm{MPa})$ & 10230 & $S_{-x}(\mathrm{MPa})$ & -600 \\
$Q_{x y}(\mathrm{MPa})$ & 3069 & $S_{+y}(\mathrm{MPa})$ & 40 \\
$Q_{s s}(\mathrm{MPa})$ & 4000 & $S_{-y}(\mathrm{MPa})$ & -100 \\
& & $S_{s}(\mathrm{MPa})$ & 40 \\
\hline
\end{tabular}

Table 1: Reference properties for the UD composite, data from [30].

pression. The stress limits used in the analyses are listed in Table $1 . S_{+x}$ and $S_{-x}$ are the tensile and compressive strengths in the fibre direction respectively, whereas $S_{+y}$ and $S_{-y}$ represent these two strengths but in the direction transverse to the fibres, hence their much lower values. $S_{s}$ represents the shear strength. In this case, the two parameters that condition the maximum load are $S_{+y}$ and $S_{s}$.

\subsection{Generation of synthetic images}

The reference speckle pattern used here is an experimental one. A speckle pattern was sprayed on a blank screen and framed with a high resolution camera $(4000 \times 2672$ pixels and 8-bit dynamic range $)$ with a pixel scale of around $0.05 \mathrm{~mm} /$ pixel. From this basic image other images with different magnification levels can be generated by cubic interpolation, see Figure 4. This procedure was employed to generate the reference image used in the deformation process.

The simulated camera has a CCD sensor of $1320 \times 1024$ pixels and 8-bit dynamic range, already leading to an undersampling of the higher resolution reference image. Since the length of the specimen will be varied in the simulation, the field of view and the magnification level are set to maximize 
the spatial resolution of the measurements and to avoid that the deformed image goes beyond the image limits. Therefore, the pixel scale $S_{p}$ writes:

$$
S_{p}=\min \left[\max \left(\frac{L^{*}}{n_{H}}, \frac{H^{*}}{n_{V}}\right), \max \left(\frac{L^{*}}{n_{V}}, \frac{H^{*}}{n_{H}}\right)\right]
$$

where $n_{H}$ and $n_{V}$ are the number of pixels in the CCD sensor in the horizontal and vertical direction, respectively. $L^{*}$ and $H^{*}$ are the length and the height of the area of measurement plus twice the maximum displacement, so that the gauge area does remains in the field of view of the camera after deformation:

$$
\left\{\begin{array}{l}
L^{*}=L+2 \max \left(\left|u_{x}\right|\right) \\
H^{*}=H+2 \max \left(\left|u_{y}\right|\right)
\end{array}\right.
$$

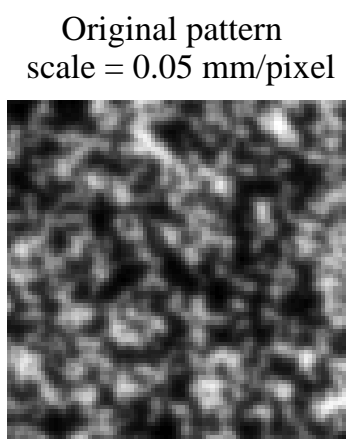

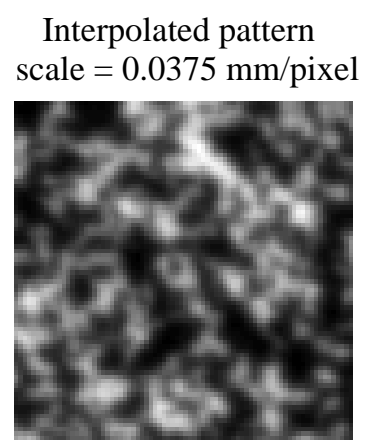

$1.5 \mathrm{~mm}$
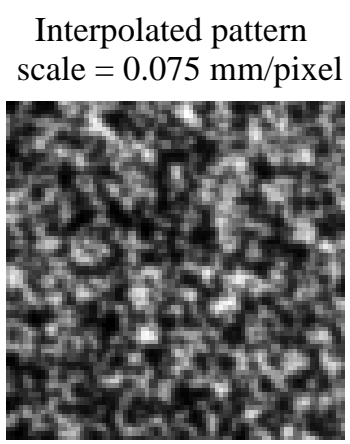

$3 \mathrm{~mm}$

Figure 4: Generation of the reference image. Starting from a real image (on the left), images with different magnification levels are obtained by grey level interpolation.

The numerical deformation process is sketched in Figure 5. Two interpolation stages are needed, named Phase I and Phase II. First, in order to map an FE field to a reference image, the discrete displacement field is 
interpolated to the integer pixel positions of the image (Phase I). Next, the reference image is deformed according to this interpolated field. This results in a grey value map at non-integer pixel positions. Accordingly, the grey values at the integer positions are obtained by interpolation of the non-integer ones, yielding a synthetically deformed image (Phase II). Phase I deals with displacement values, whereas Phase II deals with grey level values. Here all the interpolations were performed using the "TriScatteredInterp" Matlab®) function based om Delaunay triangulation, which allows to perform interpolation of a scattered dataset. A similar procedure was adopted in [31] to generate synthetic deformed speckle patterns.

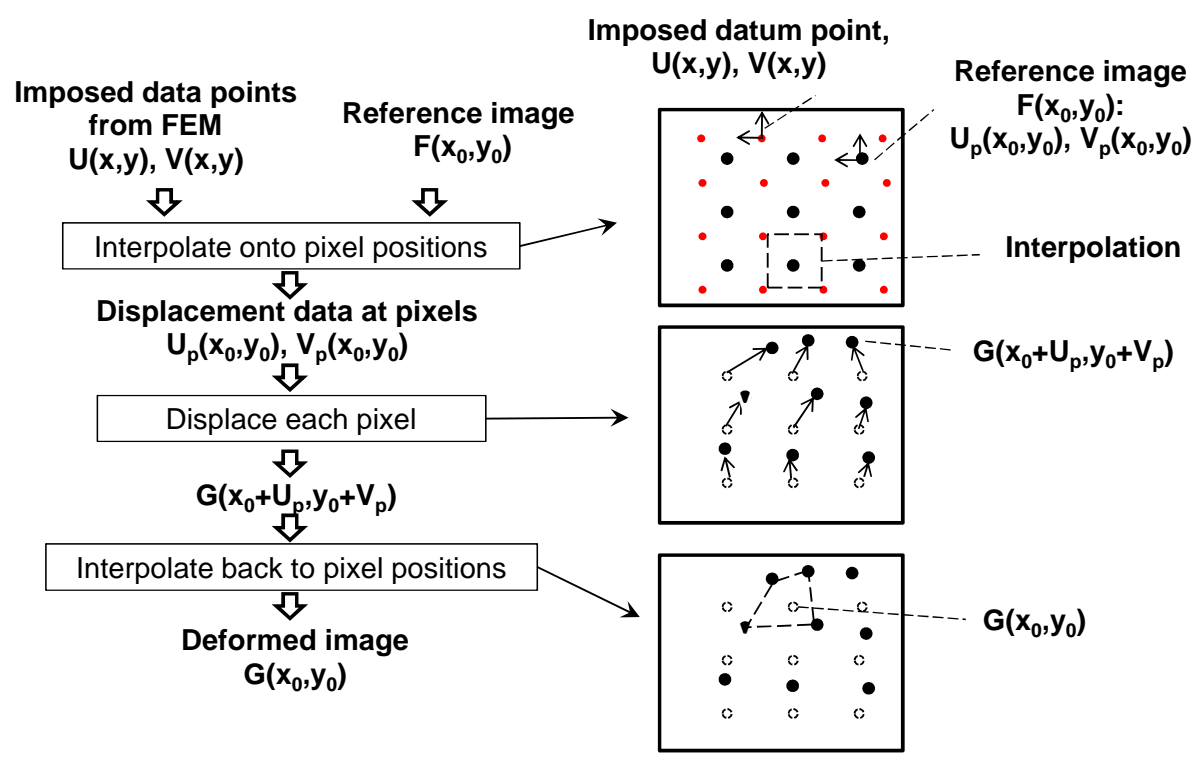

Figure 5: Construction of the numerically deformed image.

In order to reduce the numerical errors coming from the deformation routine, a pixel subsampling procedure was also implemented. The reference and deformed images were first generated at a higher resolution and then 
the final images were obtained by pixel subsampling. The procedure is illustrated in Figure 6 . In this example a $5 \times 5$ sub-pixel matrix is employed, therefore a high resolution reference image of $\left(5 n_{H}\right) \times\left(5 n_{V}\right)$ pixels with a pixel scale of $S_{p} / 5$ is created and deformed using the interpolation functions. Thus, the grey level value of each pixel of the final images is obtained as the average of the grey level of the corresponding sub-pixel matrix. Of course, the size of the sub-pixel matrix heavily influences the computational time needed to generate a synthetic image.

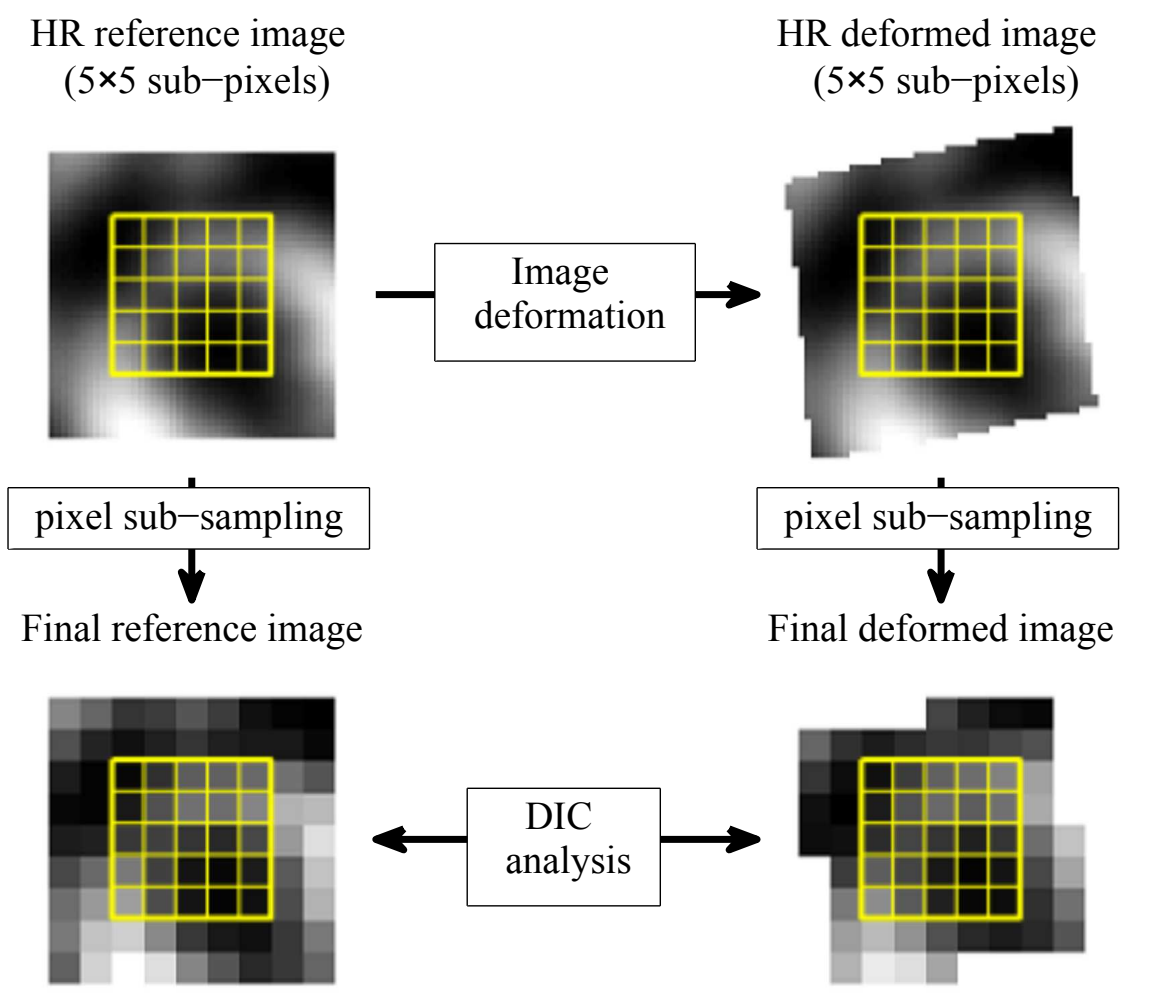

Figure 6: A pixel subsampling algorithm was used to obtain the final images where DIC is applied.

In order to validate the deformation routine, the same approach as pro- 
posed in [32] was employed. In that paper, a reference image was shifted to sub-pixel values using different deformation algorithms and the error between the imposed displacement and the one measured by DIC was computed and compared.

The same procedure was repeated here. The developed deformation routine, with and without the subsampling algorithm, was compared with the FFT approach and the "binning" technique. The last two techniques can be taken as reference, since they both represent optimal image shifting algorithms [32]. The FFT approach decomposes the image in the frequency domain and generates a shifted image through a linear phase shift. The "binning" technique uses a high resolution image to generate an image by subsampling, the displacement is obtained by shifting the subsampling matrix. Here, a $10 \times 10$ subsampling matrix is used, therefore a shift 0.1 pixel can be produced. It is worth noting that the binning technique can only be used to shift images and as such, does not require any interpolation. On the contrary, the proposed algorithm can be indifferently used to deform images of any shape with any heterogeneous displacement field.

The comparison is shown in Figure 7. If no subsampling is used, a large error is obtained compared to FFT and binning. With subsampling, even with a small sub-pixel array of $3 \times 3$, the error reduces to the same level as that of FFT and binning.

The spatial distribution of the error introduced by the image deformation algorithms and the DIC analysis is studied in Figure 8. Here the displacement and strain fields obtained with binning (reference) were compared with the ones obtained with the deformation routine, with or without subsampling. The analysis was conducted using a constant displacement of 0.3 pixels, this position corresponds to the largest error in the average displace- 


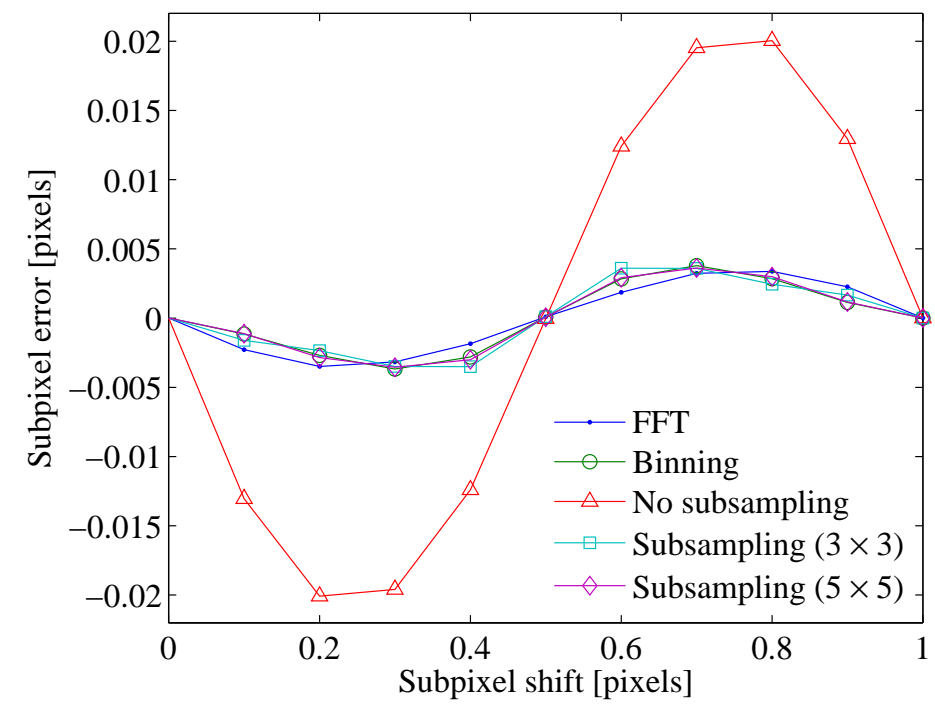

Figure 7: Comparison of different deformation algorithms.

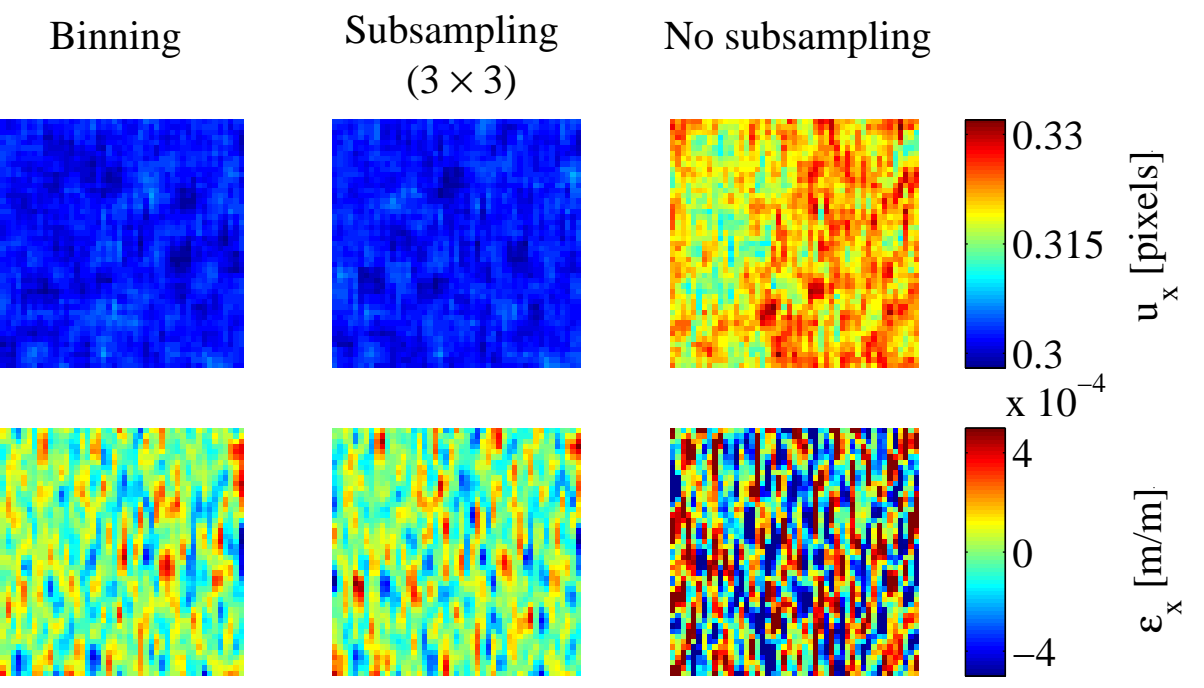

Figure 8: Comparison of the displacement and strain fields obtained with different techniques applying a shift of 0.3 pixels. 
ment observed in Figure 7. The displacement maps shows both the bias and the spatial distribution of the error. The strain maps illustrate only the spatial distribution of the error since the average strain is zero. When no subsampling is used, the displacement and strain error is larger, whereas with subsampling, the result is very similar to the one obtained with binning. This analysis validates the performance of the deformation routine. In the rest of the paper, all simulated images were obtained using a sub-pixel matrix of $3 \times 3$.

\subsection{Error evaluation}

The procedure presented above has been used to simulate the UI test under different conditions, i.e. varying the free length and the fibre orientation of the specimen, the level of noise, the smoothing intensity, the DIC parameters, etc. The total identification error Err is defined as:

$$
E r r=\sqrt{\sum_{i j} w_{i j}\left(1-\frac{Q_{i j}}{Q_{i j}^{0}}\right)^{2}} \quad \text { with } \quad i j=[x x, y y, x y, s s]
$$

where $Q_{i j}^{0}$ are the reference parameters introduced in the FE model, see Table $1, Q_{i j}$ are the parameters identified with the VFM and $w_{i j}$ is a weighting parameter that can be varied to give more or less importance to a particular stiffness component.

When noise (or other random variables) is introduced, for each configuration, several simulated experiments are repeated. In this case the error function $\operatorname{Err}$ becomes the average of the error obtained in each experiment, viz:

$$
E r r=\frac{1}{N_{e}} \sum_{k=1}^{N_{e}} E r r_{k}
$$


considering $N_{e}$ simulated experiments. This error function takes into account both the bias and the random part of the error.

Nonetheless, it reflects all the errors generated during the simulated experiment, including the artefacts caused by the numerical procedure. It is important that the latter are as small as possible.

\subsection{Error induced by FEM and interpolation}

A converged FE model is still an approximated solution of the direct problem in solid mechanics. Moreover the mesh used in the FE analysis does not coincide with the position of pixels in the image. Therefore an interpolation is necessary to obtain the displacement at each pixel position.

The interpolated displacement field used to deform the synthetic image is not an exact solution and produces an error when the VFM is used to identify the constitutive parameters. It is important to verify that such error, which represents a numerical artefact, is lower than the real errors supposed to be simulated.

A sensitivity analysis was conducted using an FE model of a specimen with length $L=30 \mathrm{~mm}$ and fibre angle $\alpha=45^{\circ}$. Similar results were found using different values of $L$ and $\alpha$. The element size of the FE model was gradually decreased from about 100 to 7 times the pixel size. The displacement values obtained from the FE model at each node have been interpolated to the pixel position of the corresponding synthetic image $(1360 \times 1024$ pixels $)$. In this case only the Phase I interpolation is considered, neither synthetic images nor optical measurement are involved.

The strain field was computed from the interpolated displacement field by finite difference and fed into the VFM for stiffness identification.

In Figure 9, the error is plotted as a function of the ratio of the pixel 


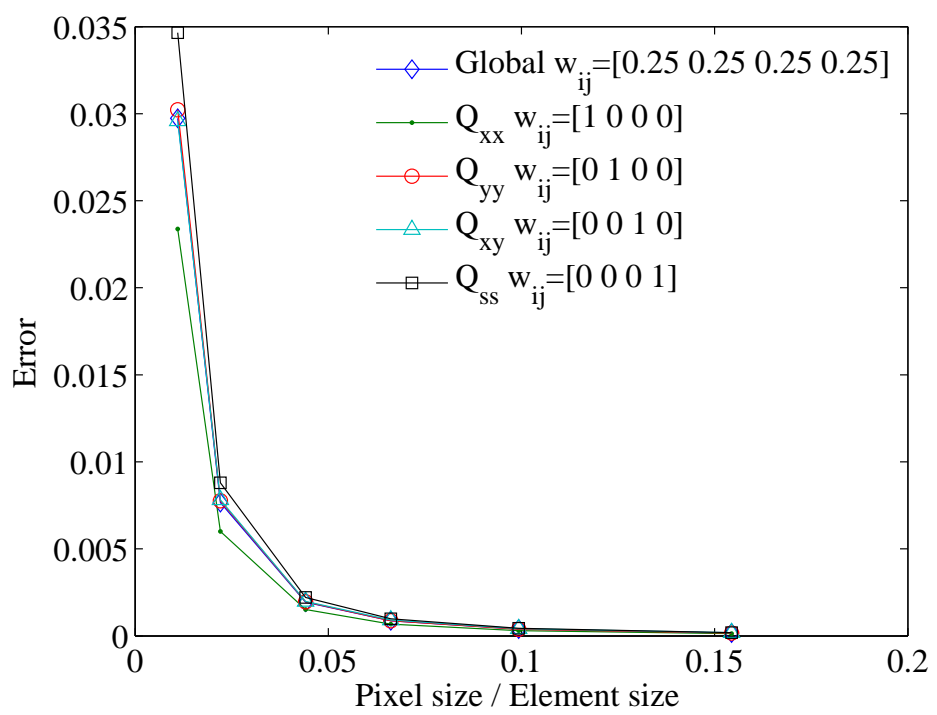

Figure 9: Influence of the strain field interpolation. Error obtained as a function of the FE mesh refinement.

size to the average element size. The weight parameter $w_{i j}$ was varied to look at the different parameters separately.

As the mesh refinement increases, a rapid drop in the error function occurs. Using an average element size 10 times larger than the pixel size, the total error is below $1 \times 10^{-3}$ for all stiffness components. This error level is much lower than the one obtained in the simulated experiments, see Figure 12 and the following. Thus it is reasonable to conclude that it will not affect the results discussed in the following sections.

\section{Results and discussions}

In this section, the simulator is used to investigate the test configuration and the DIC settings that lead to the best stiffness identification with the UI test. The following variables are studied here: the specimen parameters 
$L$ and $\alpha$, the noise level and the DIC settings (subset, VSG, step size). However, before examining this, an important point about missing data at the edges has to be reviewed.

\subsection{Effect of the measurement points at the specimen edges}

Rossi and Pierron [25] noted that in the UI test, missing data at the top and bottom edges of the specimen highly deteriorated the identification with the VFM. As illustrated in Figure 10, since the subset size is usually larger than the step size with the DIC approach, the measurement cannot be extended up to the edges of the specimen, resulting in some missing data. The larger the subset, the more missing points. It should be noted that missing data at the right and left boundaries of the field of view do not create such a problem. The issue arises with data missing at the top and bottom edges of the specimen resulting in a bias in the VFM equilibrium equation.

A simple procedure is employed here to correct this effect: the unknown values at the missing data points at the top and bottom edges have been replaced by the ones measured at the last point of the corresponding row (padding process). Even if these data are inaccurate, they are much better than missing as the transmitted stress is now reconstructed with much more fidelity. The same approach was already used in [33]. Other methods allow to extend the measurement up to the edge of the region of interest (ROI), for instance Pan et al. [34] proposed a method where the number of active pixels in the subset is automatically reduced once approaching the edge of the ROI.

In order to verify the suitability of the padding process used here, it was compared with a compensation method based on the shape functions of the 


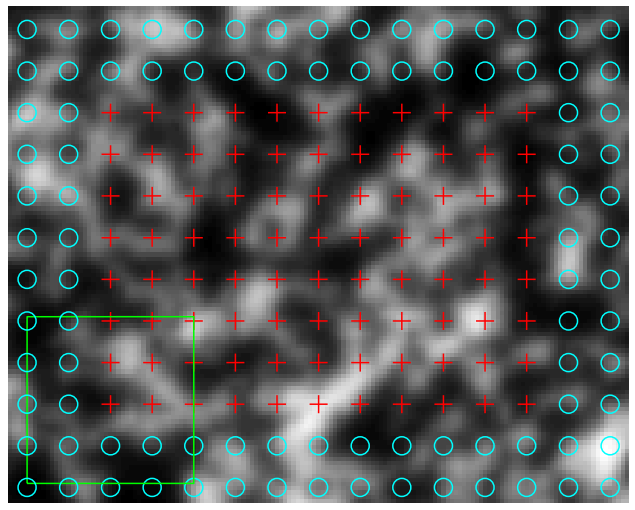

+ Measurement points Missing data Subset

Figure 10: Missing data points at the edges of the measurement area due to the subset size.

nearest evaluated subset close to the edge of the ROI. In this way, the subset keeps its full pixel capacity, no extrapolation is required, and all data points yield a consistent resolution and spatial resolution.

A simulated test was performed using a specimen with $L=30$ and $\alpha=45^{\circ}$, the subset size was varied from 11 to 44 pixels, no smoothing was applied. Three step sizes were considered: 5, 10 and 15 pixels, respectively.

Figure 11 illustrates the identification error obtained with and without the compensation methods.

Without any compensation, the error rapidly increases with the subset size and there is a strong dependency on the step size, especially for large subsets. Extending the measurment to the edges, using either of the compensation methods, the error is lower and rather independent from the subset size and the pixel step. Very similar conclusions hold for all configurations 


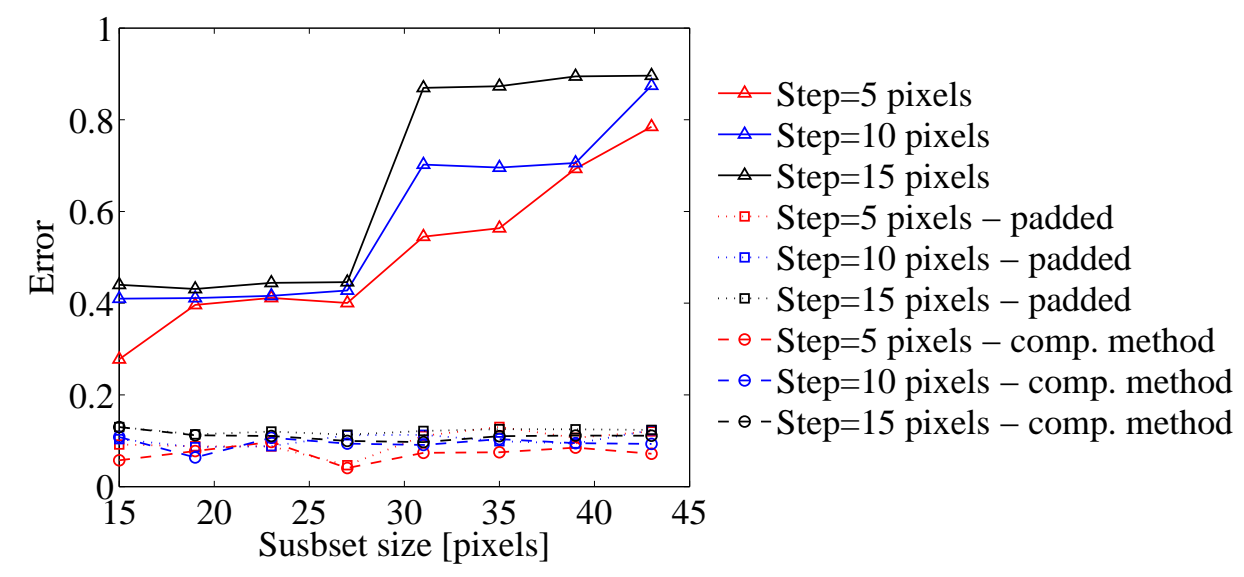

Figure 11: Influence of missing data. Identification error as function of the subset size and the step size, with and without the data padding. ( $L=30, \alpha=45^{\circ}$, no noise)

and when noise is added to the data.

More studies can be conducted on this subject, and some are already ongoing, however, for the scope of the present paper, the padding process was considered enough accurate and was used in all the following computations.

\subsection{Analysis without noise}

As a first step, noise-free images only have been considered. In this situation, the observed error indicates the bias (or systematic error) introduced by the DIC technique in the identification. The free length $L$ was varied from $10 \mathrm{~mm}$ to $60 \mathrm{~mm}$ with a step of $2 \mathrm{~mm}$, the fibre angle $\alpha$ was varied from $0^{\circ}$ to $90^{\circ}$ with a step of $5^{\circ}$. For each configuration, two 8-bit synthetic images were generated (undeformed and deformed) and the error function of Eq. 11 was evaluated.

The DIC settings were kept constant to a subset of 21 pixels and a step of 10 pixels. No smoothing was used so the VSG size is equal to the step, 

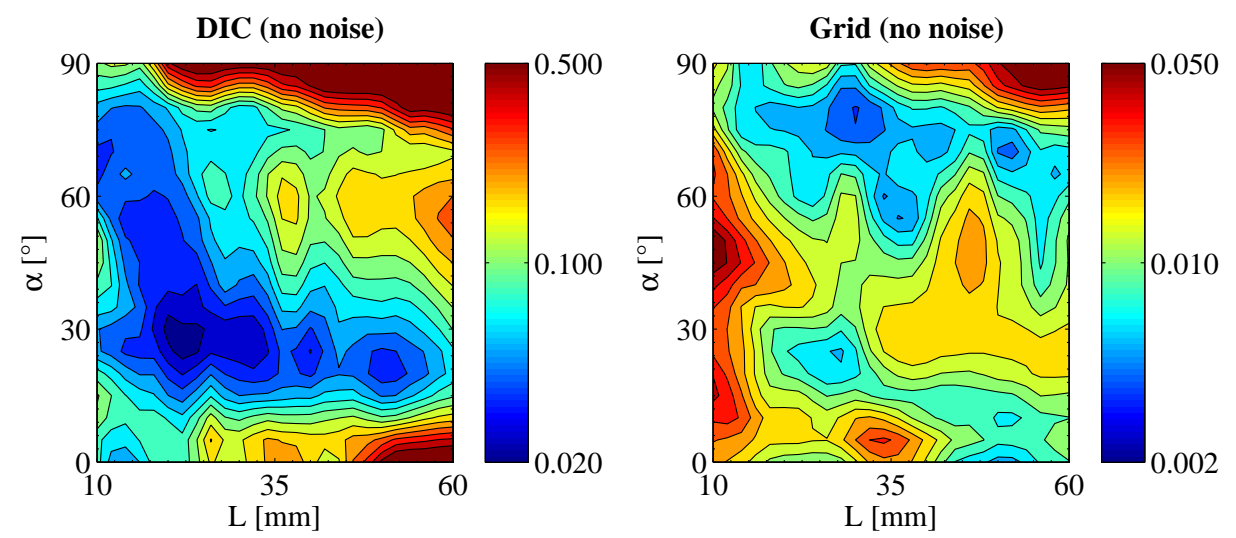

Figure 12: Error as a function of the design variables $L$ and $\alpha$.

namely 10 pixels. The influence of the DIC parameters will be studied later on.

The error obtained using DIC was compared to that from the grid method taken from a previous study [25] with the same camera resolution. The grid method analysis was performed using a sampling of 5 pixels per grid period and no smoothing.

The result is plotted in Figure 12 as a contour maps of $\operatorname{Err}$ (Eq. 11). The two axes of the graphs represent the design variables $L$ and $\alpha$, respectively. A logarithmic contour scale was used to highlight the gradient. With the grid method, the error is around ten times lower. This is not surprising since the grid method has a higher spatial resolution and is more adapted to measure low strains in large strain gradient situations.

This difference between the two measurement techniques is pointed out in Figure 13 where the strain maps generated with the simulator in the two cases are compared with the reference ones from the FE model. Only $\varepsilon_{x}$ is plotted for a specimen with $L=30 \mathrm{~mm} \alpha=45^{\circ}$, similar results are obtained for other strain components and other configurations. DIC generates a more 



Figure 13: $\varepsilon_{x}$ strain maps generated with the simulator using DIC and grid method. $\left(L=30 \mathrm{~mm} \alpha=45^{\circ}\right)$.

corrupted strain map, the error level is similar to that observed in Figure 8. The effect is highlighted in the magnification which shows the bottom left corner of the measurement area, where a high compressive strain gradient is present.

However, the higher precision of the grid method is obtained at the cost of having to bond a grid onto the specimen. Moreover, the results of Figure 12 are obtained using a perfect grid. In practice, grid defects are common and this will degrade the grid method results. This could be simulated and it will be investigated in the future.

Because of the noise generated by the technique itself, smoothing is commonly used in the strain computation with DIC and the VSG size is in general larger than the step size. A second analysis was performed using a VSG size of 141 pixels, that is a smoothing kernel of $15 \times 15$ points. The resulting strain fields are shown in Figure 14. The magnification emphasizes how the smoothing reduces the high frequency noise but also flattens out the large local strain gradients. 

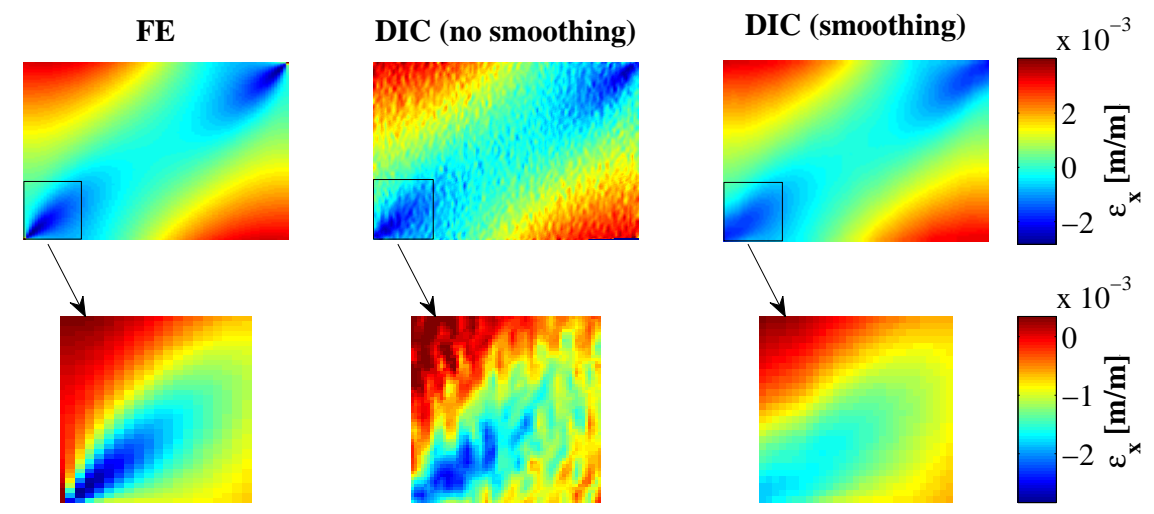

Figure 14: Strain maps generated with the simulator using DIC, without and with smoothing, i.e. $\mathrm{VSG}=141$ pixels. $\left(L=30 \mathrm{~mm} \alpha=45^{\circ}\right)$.

The effect of smoothing on the identified parameters is shown in Figure 15. It turns out that the smoothing influences both the level and the shape of the error function. With no smoothing, the minimum error is obtained for a free length of around $25-30 \mathrm{~mm}$ and a fibre orientation of $25^{\circ}-30^{\circ}$. When smoothing is introduced, a new minimum is found with $L \approx 30 \mathrm{~mm}$ and $\alpha \approx 60^{\circ}$ but with a slightly larger error than without smoothing. This is reasonable because no noise is introduced here.

\subsection{Analysis with noise}

A new analysis was conducted taking the noise into account. A Gaussian random noise was introduced in the grey level value of each synthetic image. A standard deviation of 2 grey levels was employed, this value was obtained experimentally taking two pictures of the same speckle pattern and computing the noise level as the standard deviation of the difference divided by $\sqrt{2}$.

Figure 16 illustrates the generated $\varepsilon_{x}$ strain maps when noise is intro- 

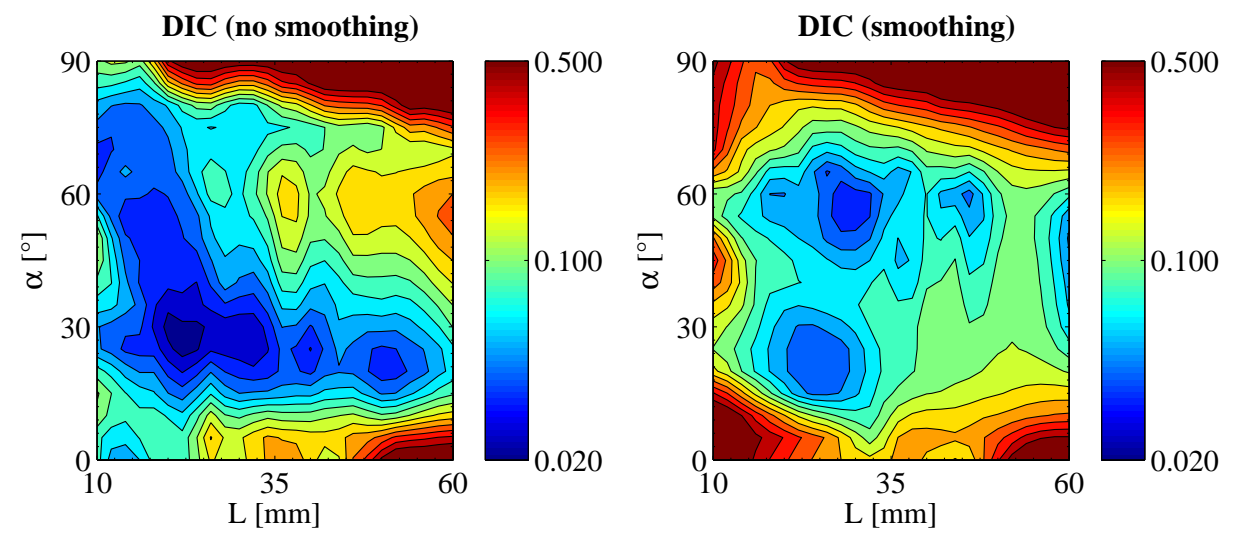

Figure 15: Error plot for DIC obtained without smoothing and with smoothing (VSG $=141$ pixels).


Figure 16: Strain maps generated with the simulator introducing noise. DIC with no smoothing, DIC with VSG = 141 pixels and grid method without smoothing are compared. $\left(L=30 \mathrm{~mm} \alpha=45^{\circ}\right)$. 

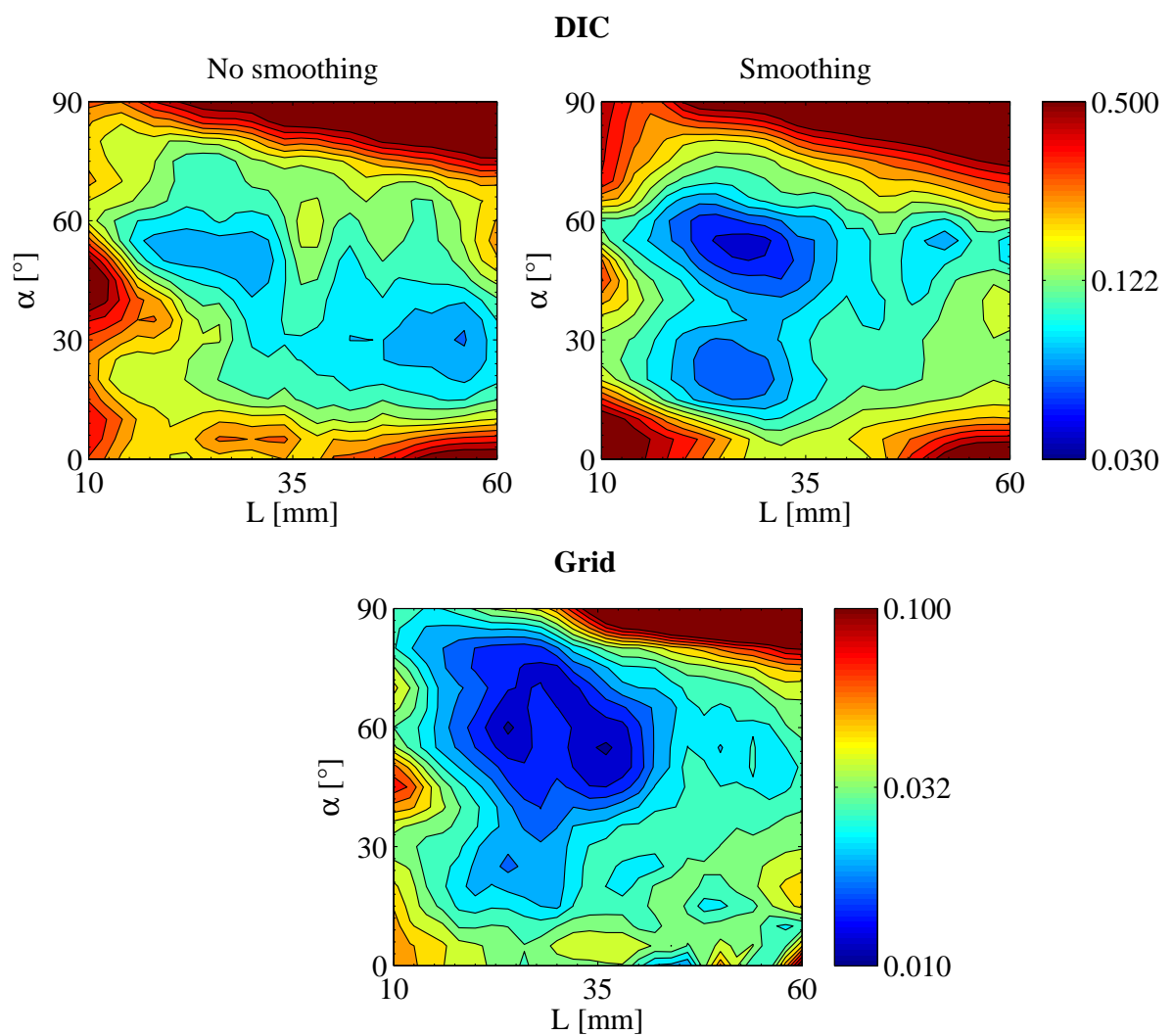

Figure 17: Comparison between the error obtained using DIC, without and with smoothing $(\mathrm{VSG}=141$ pixels), and the grid method without smoothing [25]. 8-bit images are used with 2 grey levels noise standard deviation. 
duced. The effect of noise is clearly visible when no smoothing is performed. The spatial frequency of the noise is lower with DIC than with grid, this is due to the lower spatial resolution of DIC. When smoothing is introduced, with a VSG size of 141 pixels, the high frequency noise is filtered out and the strain map looks similar to the one of Figure 14.

The effect of noise in the identification is reported in Figure 17. This time, the total error Err of Eq. 12 was evaluated considering 20 repetitions for each configuration. Each virtual experiment is indeed different because of the random noise introduced into the data. The error obtained using DIC with and without smoothing was compared to that from the grid method. The same grey level noise was used for all cases.

Looking at the results for DIC, again the smoothing influences the shape of the error function. In this case, the best result was obtained with smoothing. This makes sense because this time noise was introduced in the generated images. It is interesting to observe that the error obtained with noise is very similar to the one without noise if smoothing is introduced. This shows that a VSG size of 141 pixels filters out the effect of random noise and that the remaining error mainly comes from the reconstruction error.

Looking at the grid method, when noise is introduced, a larger error is obtained with the minimum value that goes from 0.002 of Figure 12 to 0.01 .

Qualitatively, the error map of the grid method looks very similar to that of DIC with smoothing. The configuration with the lowest error is similar in both cases, i.e. $L=30 \mathrm{~mm}$ and $\alpha=50^{\circ}-60^{\circ}$. In fact, as already demonstrated in [25], $30 \mathrm{~mm}$ corresponds to an aspect ratio of $S$ similar to that of the CCD sensor, showing the importance of the spatial resolution. Looking at the numerical value in the optimal configuration, the error for DIC is around three times larger than for the grid method. In non 
optimal configurations, the difference increases and the identification error using DIC is approximately five to six times larger than for the grid method.

In order to investigate the similarity between the error achieved with DIC and grid method, further analysis was conducted looking at the identification of each single stiffness component. Figure 18 shows the error computed separately for each $Q_{i j}$ by suitably setting the weighting parameter $w_{i j}$ in Eq. 11 to 1 for a given stiffness component and 0 for the others. For DIC, the analysis with smoothing was considered. For each parameter, the same considerations as for Figure 17 can be made: using DIC, the average error is larger, however, qualitatively, the four maps look similar.

\subsection{Influence of DIC settings on noise}

In this section, the interaction between DIC parameters and noise is evaluated. A single configuration is studied with $L=30 \mathrm{~mm}$ and $\alpha=55^{\circ}$, which represents the optimum configuration of Figure 17. The same amount of noise of 2 grey level is used.

In Figure 19, the error was evaluated as a function of the subset and the VSG size. It is possible to obtain an optimal setting with a subset of 23 pixels and a VSG of 171 pixels. With respect to the configuration used in the previous section (subset $=21$ pixels, $\mathrm{VSG}=141$ pixels), a further improvement (15-20\%) is obtained with the error reducing from 0.03 to 0.025. Looking at the contour map, the VSG is the parameter that mostly influences the error.

To look at the effect of the noise level, the analysis was repeated using a standard deviation of noise of 15 grey levels. This value is exaggerated compared to actual experiments and is used here only as for the sake of sensitivity analysis. The results are shown on Figure 20. As expected the 

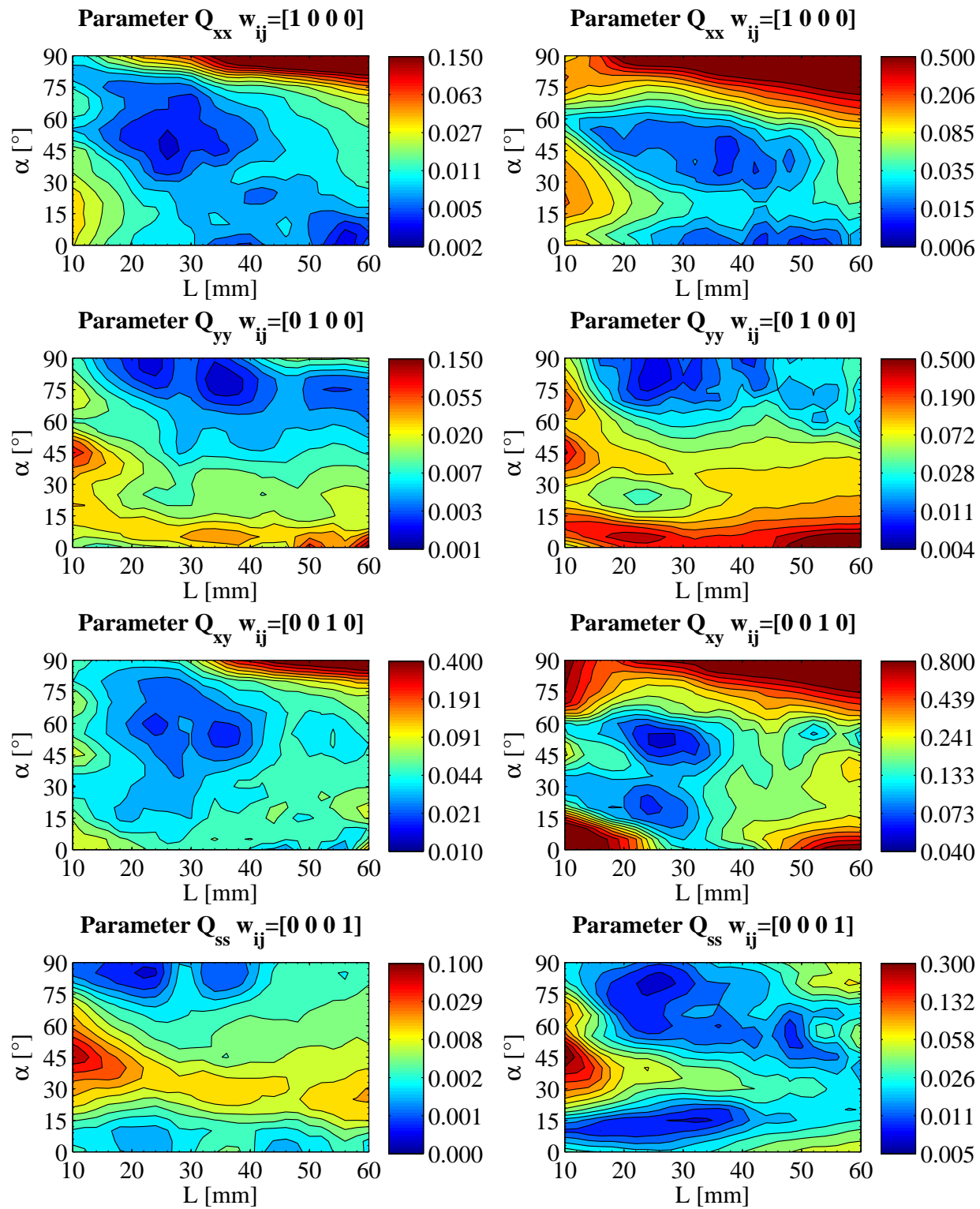

(a) Grid Method



(b) DIC

Figure 18: Error function evaluated separately for the different parameters. DIC and grid method are compared. 




Figure 19: Influence of the subset and the VSG size on the identification error. Standard deviation of noise: 2 grey levels.

value of the error is much higher. The minimum error is obtained with a VSG of 321 pixels, showing that in this case more smoothing is favourable, as one would expect. The best subset size is 15 pixels, which is a bit surprising. A possible explanation is that a small subset size and a high level of smoothing obtained by VSG allow to better describe the strain field close to the edges.

The present results show that the optical measurement parameters have an important effect on the quality of the final stiffness identification. Moreover, the choice of the different parameters (subset size, smoothing kernel etc.) can be performed more rationally if such a simulator is available. For a given test configuration, it should be possible to employ this tool to provide realistic confidence intervals for identified material parameters. This is very important if such new approaches are to become test standards in the near future. Finally, this tool can also be employed to design and optimize 




Figure 20: Influence of the subset and the VSG size on the identification error. Standard deviation of noise: 15 grey levels.

new test configurations with a rational procedure. It opens up very wide prospects in the design of novel test methods.

\subsection{Influence of the step size}

In the previous analyses, the step size was kept constant to 10 pixels, in this section, a study was conducted to investigate in more details its effect. In Figure 21, the identification error was evaluated varying the step size from 2 to 20 pixels, keeping the VSG size constant to 81 pixels. The adopted specimen configuration was $L=30 \mathrm{~mm}$ and $\alpha=55^{\circ}$. First no noise was added, and three different subsets were considered: 21, 31, and 41 pixels. Then, using a subset of 21 pixels, the same analysis was conducted introducing noise with a standard deviation of 2 pixels.

It turns out that the step size has an impact on the identification error, 

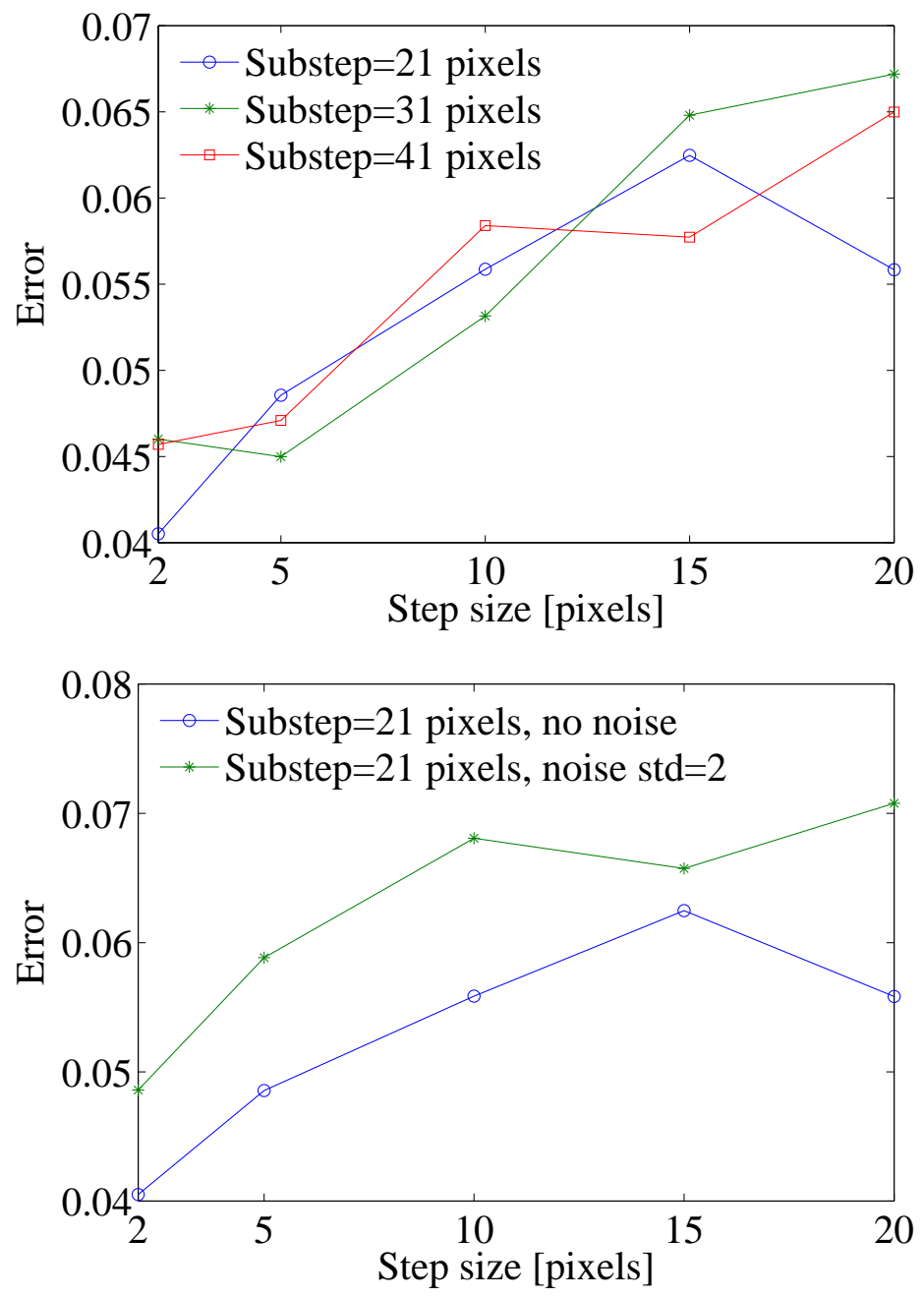

Figure 21: Influence of the step size on identification error, without noise and with noise. 
and a small step size can improve the identification quality. The same trend was obtained when noise is added to the synthetic images. This suggests that the step size influences the identification error when high gradients have to be measured.

\subsection{Influence of the grey level range}

A final study was conducted to verify the influence of the grey level encoding. Synthetic images were create using 16-bit dynamic range, the speckle pattern is simply scaled from the 8-bit one, used in the previous analysis. A specimen configuration, with $L=30 \mathrm{~mm}$ and $\alpha=55^{\circ}$, was used. The following DIC parameters were used: subset $=21$ pixels, step $=10$ pixels, $V S G=141$ pixels; similar results are obtained using different configurations.

Figure 22 shows that, if the ratio of noise standard deviation to the grey level range is the same, the use of 8-bit or 16-bit images gives the same result in terms of identification error. Of course, a large dynamic range gives more flexibility on the histogram, and is beneficial, especially in case of poor illumination.

\section{Conclusion}

A general procedure to numerically simulate an actual test, i.e. the UI test, has been proposed. The procedure starts from an FE model of the test and generates synthetic images deformed from a pre-existent reference image. The synthetic images aim to simulate a real acquisition performed during an experimental test. After the simulation process, the displacement and strain fields are computed using DIC and then the VFM is applied to identify the material properties. An error function is used to assess the 


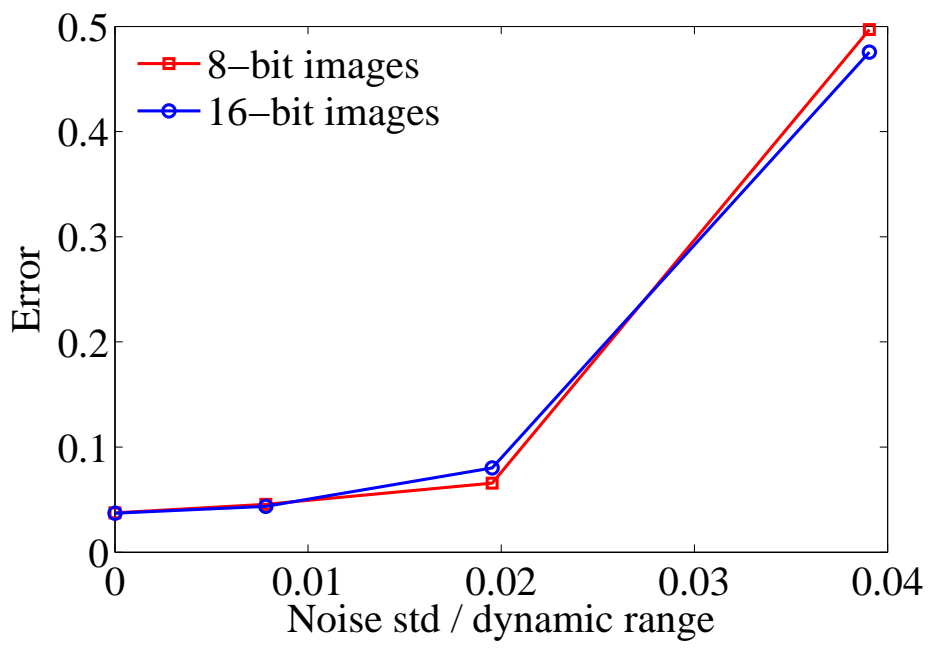

Figure 22: Influence of the dynamic range on identification error.

quality of the identification procedure. The numerical procedure was designed to use real speckle pattern pictures as reference images. In order to avoid numerical artefacts at low displacements, a subsampling algorithm was employed.

The following concluding considerations can be made:

- the identification with the UI test is highly influenced by missing data at the edges. The adopted DIC technique produces missing data because of the subset size. A simple procedure is proposed to correct the data by padding and a remarkable improvement is obtained in the identification.

- The performance of the DIC was compared with the grid method. When noise is introduced, using an optimal configuration, the error obtained with DIC is around three times larger than the one obtained with the grid method, as expected from the better spatial resolution 
of the latter, at the cost of having to prepare the specimen surface with a grid pattern. On the other hand, in the simulation of the grid method, the used grid did not have any defect (missing bits of grid, non-uniform grid pitch), which, in actual tests, can increase the error.

- The procedure was used to evaluate the sensitivity of the identification routine to the noise level. According to the level of noise, the developed procedure provides a rational route to the selection of good DIC parameter settings. The VSG size is the most influential among those studied.

In future work, the simulator can be used to design test configurations which maximize the performances of DIC and VFM. Moreover, other sources of error could be introduced like for instance misalignment of the specimen or out-of-plane movements during the test. In order to have a complete validation of the procedure, other experimental tests should be simulated and the numerical experiments should be compared with actual ones.

\section{Acknowledgements}

Professor Pierron gratefully acknowledges support from the Royal Society and the Wolfson Foundation through a Royal Society Wolfson Research Merit Award. 


\section{References}

[1] Sutton, M.A., Orteu, J.-J. and Schreier, H.W. (2009) Image correlation for shape, motion and deformation measurements. Springer New-York.

[2] Cooreman, S., Lecompte, D., Sol, H., Vantomme, J. and Debruyne, C. (2007) Elasto-plastic material parameter identification by inverse methods: Calculation of the sensitivity matrix. Int. J. Solids Struct. 44, $4329-4341$.

[3] Lecompte, D., Smits, A., Sol, H., Vantomme, J. and Van Hemelrijck, D. (2007) Mixed numerical-experimental technique for orthotropic parameter identification using biaxial tensile tests on cruciform specimens. Int. J. Solids. Struct. 44(5), 1643-1656.

[4] Le Magorou, L., Bos, F. and Rouger, F. (2002) Identification of constitutive laws for wood-based panels by means of an inverse method. Compos. Sci. Technol. 62, 591-596.

[5] Kajberg, J. and Lindkvist, G. (2004) Characterisation of materials subjected to large strains by inverse modelling based on in-plane displacement fields. Int. J. Solids Struct. 41, 3439-3459.

[6] Coppieters, S., Cooreman, S., Sol, H., Van Houtte, P. and Debruyne, D. (2011) Identification of the post-necking hardening behaviour of sheet metal by comparison of the internal and external work in the necking zone. J. Mater. Process. Tech. 211(3), 545-552.

[7] Latourte, F. ,Chrysochoos, A., Pagano, S. and Wattrisse, B. (2008) Elastoplastic behavior identification for heterogeneous loadings and materials. Exp. Mech. 48(4), 435-449. 
[8] Geymonat, G. and Pagano, S. (2003) Identification of mechanical properties by displacement field measurement: a variational approach. Meccanica $38,535-545$.

[9] Claire, D., Hild, F. and Roux, S. (2004) A finite element formulation to identify damage fields: the equilibrium gap method. Int. J. Numer. Meth. Eng. 61, 189-208.

[10] Bui, H. D., Constantinescu, A. and Maigre, H. (2004) Numerical identification of linear cracks in 2D elastodynamics using the instantaneous reciprocity gap. Inverse Probl. 20, 993-1001.

[11] Pierron, F. and Grédiac, M. (2012) The Virtual Fields Method. Springer New York.

[12] Giraudeau, A. and Pierron, F. (2005) Identification of stiffness and damping properties of thin isotropic vibrating plates using the virtual fields method. theory and simulations. J. Sound. Vib. 284, 757-781.

[13] Grédiac, M. and Pierron, F. (2006) Applying the virtual fields method to the identification of elasto-plastic constitutive parameters. Int. J. Plasticity 22, 602-627.

[14] Rossi, M. and Pierron, F. (2012) Identification of plastic constitutive parameters at large deformations from three dimensional displacement fields. Comput. Mech. 49, 53-71.

[15] X. Dai and H. Xie. Constitutive parameter identification of 3d printing material based on the virtual fields method. Measurement, 59:38-43, 2014 . 
[16] Pierron, F., Vert, G., Burguete, R., Avril, S., Rotinat, R. and Wisnom M. (2007) Identification of the orthotropic elastic stiffnesses of composites with the virtual fields method: sensitivity study and experimental validation. Strain 43(3), 250-259.

[17] Bornert, M., Brémand, F., Doumalin, P., Dupré, J.-C., Fazzini, M., Grédiac, M., Hild, F., Mistou, S., Molimard, J., Orteu, J.-J., Robert, J.-J., Surrel, Y., Vacher, P. and Wattrisse, B. (2009) Assessment of digital image correlation measurement errors: methodology and results. Exp. Mech. 49, 353-370.

[18] Lava, P., Cooreman, S., Coppieters, S., De Strycker, M. and Debruyne, D. (2009) Assessment of measuring errors in DIC using deformation fields generated by plastic FEA. Opt. Laser Eng. 47, 747-753.

[19] Lava, P., Cooreman, S. and Debruyne, D. (2010) Study of systematic errors in strain fields obtained via DIC using heterogeneous deformation generated by plastic FEA. Opt. Laser. Eng. 48(4), 457-468.

[20] Patterson, E.A., Hack, E., Brailly, P., Burguete, R.L., Saleem, Q., Siebert, T., Tomlinson, R.A. and Whelan, M.P. (2007) Calibration and evaluation of optical systems for full-field strain measurement. Opt. Laser. Eng. 45(5), 550-564.

[21] Patterson, E., Brailly, P., Burguete, R., Hack, E., Siebert T. and Whelan, M. (2007) A challenge for high-performance full-field strain measurement systems. Strain 43(3), 167-180.

[22] Wattrisse, B., Chrysochoos, A., Muracciole, J.-M. and Némoz- 
Gaillard, M. (2001) Analysis of strain localization during tensile tests by digital image correlation. Exp. Mech. 41(1), 29-39.

[23] Wang, Y., Lava, P., Coppieters, S., De Strycker, M., Van Houtte, P. and Debruyne, D. (2012) Investigation of the uncertainty of DIC under heterogeneous strain states with numerical tests. Strain 48(6), 453-462.

[24] Schreier, H.W. and Sutton, M.A. (2002) Systematic errors in digital image correlation due to undermatched subset shape functions. Exp. Mech. 42(3), 303-310.

[25] Rossi, M. and Pierron, F. (2012) On the use of simulated experiments in designing tests for material characterization from full-field measurements. Int. J. Solids Struct. 49, 420-435.

[26] Pierron, F. and Grédiac, M. (2000) Identification of the throughthickness moduli of thick composites from whole-field measurements using the Iosipescu fixture: theory and simulations. Compos. Par. AAppl. S. 31, 309-318.

[27] Avril, S., Ferrier, E., Hamelin, E., Surrel, Y. and Vautrin, A. (2004) A full-field optical method for the experimental analysis of reinforced concrete beams repaired with composites. Compos. Par. A-Appl. S. 35, $873-884$.

[28] Avril, S., Vautrin, A. and Surrel, Y. (2004) Grid method: Application to the characterization of cracks. Exp. Mech. 44(1), 37-43.

[29] Orteu, J.-J., Garcia, D., Robert, L. and Bugarin, F. (2006) A speckletexture image generator. In Proc. Speckle'06 Int. Conf., Nîmes, France. 
[30] Tsai, H. W., and Hahn, H. T. (1980) Introduction to composite materials. Techonomic Publishing Company, Inc.

[31] Lecompte, D., Smits, A., Bossuyt, S., Sol, H., Vantomme, J., Van Hemelrijck, D. and Habraken, A.M. (2006) Quality assessment of speckle patterns for digital image correlation. Opt. Laser. Eng. 44(11), $1132-1145$.

[32] Reu, P. L. (2001) Experimental and numerical methods for exact subpixel shifting. Exp. Mech. 51, 443-452.

[33] P. Wang, F. Pierron, and O.T. Thomsen. Identification of material parameters of PVC foams using digital image correlation and the virtual fields method. Exp, Mech,, 53(6):1001-1015, 2013.

[34] B. Pan, Z. Wang, and Z. Lu. Genuine full-field deformation measurement of an object with complex shape using reliability-guided digital image correlation. Opt. Express, 18(2):1011-1023, 2010. 